\title{
CONFORMAL MAPS WITH LEAST DISTORTION
}

\author{
H. G. HELFENSTEIN
}

1. Introduction. Our problem is related to the construction of geographical maps as follows. The reason for using conformal geographical maps is that the scale (viz., the ratio of two corresponding line-elements) does not depend on the direction of these line-elements. In an ideal map the scale, being responsible for the preservation of shape, would also be independent of the points where the line-elements begin. Since this is impossible, except for developable surfaces, one tries to construct maps whose scales are "as constant as possible." According to the precise meaning of this expression several "best" maps are possible. Tchebycheff (1) studied the case in which the maximum deviation of the scale from a certain constant is minimized. We shall consider here the problem of minimizing the mean quadratic deviation of the scale from a constant. In order to linearize this problem we have to use, as Tchebycheff did also, the logarithm of the scale instead of the scale itself. We prove the existence of a best map in this sense for a simply connected domain on an arbitrary surface. In addition we give some explicit formulae for computing it.

The definition of the mean quadratic error depends on the choice of the parameters on the surface. We use a "normal" system of isothermic coordinates mapping the given surface on the interior of the unit circle of a complex plane. Then our problem reduces to that of the best approximation of a given function by harmonic functions.

2. Notation. Let $D$ be a finite, simply connected domain with more than one boundary point on a surface $S$ which is sufficiently "smooth." Assume that we can find an isothermic system of parameters mapping $D$ on a plane schlicht domain $D^{\prime}$ which in turn can be transformed conformally on the interior of the unit circle $U$ of a complex $z$-plane. If $z=x+i y$, then $x$ and $y$ are again isothermic parameters on $D$ which we call a normal system. It is uniquely determined if we let an arbitrary point $M$ of $D$ correspond to the point $z=0$ and an arbitrary direction through $M$ to the direction of the positive $x$-axis. The line-element of $D$ becomes in the $x, y$ system:

$$
d s=\frac{1}{\gamma(z)} \sqrt{ }\left(d x^{2}+d y^{2}\right)=\frac{|d z|}{\gamma(z)},
$$

where $\gamma(z)$ is a real positive function. Every other conformal map of $D$ can be obtained as a regular analytic function $w=u+i v=f(z)$ defined in $U$ whose derivative must not vanish. In order to fix the image of $U$ with respect to congruent transformations we require that

$$
f(0)=0, \quad \arg f^{\prime}(0)=0 .
$$

Received August 16, 1954; in revised form January 28, 1955. 
We can prescribe also the value of the scale in the fixed point $M$ :

$$
\left|f^{\prime}(0)\right|=\frac{\mu}{\gamma(0)} .
$$

The distortion in a point $x, y$ now becomes:

$$
m=\frac{|d w|}{d s}=\left|f^{\prime}(z)\right| \frac{|d z|}{d s}=\left|f^{\prime}(z)\right| \gamma(z) .
$$

Since $f^{\prime}(z) \neq 0$ and $\gamma>0$ we can put

$$
\begin{aligned}
\Re \log f^{\prime}(z) & =\log \left|f^{\prime}(z)\right|=\phi(x, y), \\
\log \gamma(z) & =\varphi(z),
\end{aligned}
$$

whereupon (3) takes the form

$$
m=e^{\phi+\varphi},
$$

$\phi$ being a harmonic function in $U$. The smoothness of $D$ is now specified as follows: The function $\varphi(x, y)$, which is determined by $D$, shall be at least twice differentiable with the derivatives bounded in $|z|<1$.

If $m$ is to be as constant as possible in $U$ then the same must be true for $\phi+\varphi$. We shall therefore determine a harmonic function $\phi$ and a constant $C$ such that

$$
I=\int_{U} \int(\phi+\varphi-C)^{2} d x d y
$$

becomes a minimum subject to the above mentioned side conditions for $f(z)$. In particular (2) reads as follows:

$$
\log \left|f^{\prime}(0)\right|=\phi(0)=\log \frac{\mu}{\gamma(0)} .
$$

3. Existence theorem. If $\varphi(z)=\varphi(R, \theta)$ belongs to the class $C^{(2)}$ in $|z| \leqslant 1$, (a) There exists a function $\phi(R, \theta)$ harmonic in $|z|<1$ with

$$
\phi(0)=\log \frac{\mu}{\gamma(0)}
$$

and a constant $C$ which minimize the integral (6).

(b) If

$$
\phi(R, \theta)-C=\sum_{k=-\infty}^{\infty} b_{k}(R) e^{i k \theta}
$$

then $b_{k}=A_{k} R^{k}$,

where

$$
A_{k}=-\frac{k+1}{\pi} \int_{0}^{1} \int_{0}^{2 \pi} \varphi(R, \theta)\left(R e^{-i \theta}\right)^{k} R d R d \theta,
$$

and

$$
C=\log \frac{\mu}{\gamma(0)}-A_{0}
$$


(c) The mapping function subject to conditions (1) and (2) is given by

$$
f(z)=\frac{\mu}{\gamma(0)} \int_{0}^{z} \exp \left(2 \sum_{k=1}^{\infty} A_{k} t^{k}\right) d t .
$$

Proof. Introduce polar coordinates by

$$
x=R \cos \theta, \quad y=R \sin \theta,
$$

and expand in Fourier Series

$$
\begin{gathered}
\varphi=\sum_{k=-\infty}^{+\infty} a_{k}(R) e^{i k \theta}, \quad a_{k}(R)=\frac{1}{2 \pi} \int_{0}^{2 \pi} \varphi e^{-i k \theta} d \theta, \\
k=0, \pm 1, \pm 2, \ldots, \\
\phi+\varphi-C=\sum_{k=-\infty}^{+\infty} C_{k}(R) e^{i k \theta} .
\end{gathered}
$$

Since these functions are real we have

$$
a_{-k}=\bar{a}_{k}, \quad C_{-k}=\bar{C}_{k}
$$

for every $k$; since they are one-valued we get

$$
a_{k}(0)=0, \quad C_{k}(0)=0
$$

for $k \neq 0$, and

$$
a_{0}(0)=\varphi(0), \quad C_{0}(0)=\phi(0)+\varphi(0)-C .
$$

Combining (8) and (9) we obtain

$$
\phi=\sum_{-\infty}^{+\infty}\left[C_{k}(R)-a_{k}(R)\right] e^{i k \theta}+C,
$$

and integrating the condition $\nabla^{2} \phi=0$ under the side conditions (10)-(12), we find, for $k \geqslant 0$,

$$
C_{k}(R)=A_{k} R^{k}+a_{k}(R), \quad C_{-k}(R)=\overline{C_{k}(R)}
$$

where the sequence of the constants of integration $A_{k}(k=0,1,2, \ldots)$ is to be determined by our minimum postulate.

We use now the following identity which is true for every pair of complex numbers $P$ and $Q$ :

$$
|P|^{2}+P \bar{Q}+\bar{P} Q=|P+Q|^{2}-|Q|^{2} .
$$

Applying it to

$$
P=\frac{A_{k}}{\sqrt{ } 2(k+1)}, \quad Q=\sqrt{ } 2(k+1) \int_{0}^{1} a_{k}(R) R^{k+1} d R
$$

we obtain from (14):

$$
\begin{aligned}
\int_{0}^{1}\left|C_{k}(R)\right|^{2} R d R & =\left|\frac{A_{k}}{\sqrt{ } 2(k+1)}+\sqrt{ } 2(k+1) \int_{0}^{1} a_{k}(R) R^{k+1} d R\right|^{2} \\
& -2(k+1)\left|\int_{0}^{1} a_{k}(R) R^{k+1} d R\right|^{2}+\int_{0}^{1}\left|a_{k}(R)^{2}\right| R d R .
\end{aligned}
$$


The completeness relation yields now for the integral (6):

$$
I=2 \pi \int_{0}^{1}\left|C_{0}(R)\right|^{2} R d R+4 \pi \sum_{k=1}^{\infty} \int_{0}^{1}\left|C_{k}(R)\right|^{2} R d R,
$$

where the interchange of summation and integration will be justified later. Taking into account (15), (16) assumes the form:

$$
\begin{aligned}
I= & 2 \pi\left|\frac{A_{0}}{\sqrt{ } 2}+\sqrt{ } 2 \int_{0}^{1} a_{0}(R) R d R\right|^{2}-4 \pi\left|\int_{0}^{1} a_{0}(R) R d R\right|^{2} \\
& +2 \pi \int_{0}^{1} a_{0}^{2}(R) R d R \\
& +4 \pi \sum_{k=1}^{\infty}\left\{\left|\frac{A_{k}}{\sqrt{ } 2(k+1)}+\sqrt{ } 2(k+1) \int_{0}^{1} a_{k}(R) R^{k+1} d R\right|^{2}\right. \\
& \left.-2(k+1)\left|\int_{0}^{1} a_{k}(R) R^{k+1} d R\right|^{2}+\int_{0}^{1}\left|a_{k}(R)\right|^{2} R d R\right\} .
\end{aligned}
$$

Here the unknown constants appear only within squares. Consequently $I$ assumes its minimum for the following values of the constants:

$$
A_{k}=-2(k+1) \int_{0}^{1} a_{k}(R) R^{k+1} d R, \quad k=0,1,2, \ldots,
$$

or, according to (8):

$$
A_{k}=-\frac{k+1}{\pi} \int_{0}^{1} \int_{0}^{2 \pi} \varphi(R, \theta)\left(R e^{-i \theta}\right)^{k} R d R d \theta .
$$

Combining (11), (12), and (14) one recognizes that the constant $C$ is also completely determined:

$$
C=\phi(0)-A_{0}=\log \frac{\mu}{\gamma(0)}+\frac{1}{\pi} \int_{0}^{1} \int_{0}^{2 \pi} \varphi(R, \theta) R d R d \theta .
$$

From equations (13) and (18) one concludes that

$$
\phi=\log \frac{\mu}{\gamma(0)}+2 \sum_{k=1}^{\infty} R^{k}\left(A_{k}^{\prime} \cos k \theta-A_{k}^{\prime \prime} \sin k \theta\right),
$$

where

$$
A_{k}^{\prime}=\Re A_{k} \text { and } A_{k}^{\prime \prime}=\Im A_{k} .
$$

From this expression one finds the conjugate harmonic function in the usual way, taking into account (1) and (2) in order to determine the constants of integration. This finally leads to

$$
f(z)=\frac{\mu}{\gamma(0)} \int_{0}^{z} \exp \left(2 \sum_{1}^{\infty} A_{k} t^{k}\right) d t .
$$

4. Convergence. The convergence of the power series

$$
\sum_{1}^{\infty} A_{k} z^{k} \quad \text { in }|z|<1
$$

follows from known estimates of the Fourier coefficients. If $\varphi$ is $h-1$ times differentiable with respect to $\theta$ and possesses a piecewise continuous derivative 
of order $h$ with a bound $M_{h}$, then $h$-times repeated integration by parts of (8) yields:

$$
\left|a_{k}(R)\right| \leqslant M_{h} / k^{h} \text {. }
$$

Combining this with (18) we have

$$
\left|A_{k}\right|<2 M_{h} / k^{h} \text {. }
$$

For $|z| \leqslant r_{0}<1$ the series $\sum A_{k} z^{k}$ is therefore majorized by $\sum 2 M_{0} r_{0}{ }^{k}$ which is independent of $z$. The boundness of $\varphi$ is thus seen to be sufficient for the circle of convergence of $(21)$ to contain $U$.

If $h=2$ (as previously assumed) then (21) is also uniformly convergent on the boundary of $U$ (which of course cannot prevent the function $f(z)$ from possibly having a singularity on the boundary).

From (14) and (22) with $h=1$ one concludes the uniform convergence of

$$
\sum_{1}^{\infty}\left|C_{k}(R)\right|^{2}, \quad 0 \leqslant R \leqslant 1,
$$

which has been used in the interchange of summation and integration in (16).

5. Mean value theorem. For every integer $k \geqslant 0$ the mean value of the function $\varphi(R, \theta) R^{k} e^{ \pm i k \theta}$ on the unit circle $|z|<1$ is numerically equal but opposite in sign to the mean value of $\phi(R, \theta) R^{k} e^{ \pm i k \theta}$.

Proof. Equation (13) yields:

$$
C_{k}(R)-a_{k}(R)=\frac{1}{2 \pi} \int_{0}^{2 \pi} \phi(R, \theta) e^{-i k \theta} d \theta, \quad k=0, \pm 1, \pm 2, \ldots
$$

hence we have from (14) and (19)

$$
A_{k} R^{k}=-R^{k} \cdot \frac{k+1}{\pi} \int_{0}^{1} \int_{0}^{2 \pi} \varphi(r, \theta) r^{k} e^{-i k \theta} r d r d \theta=\frac{1}{2 \pi} \int_{0}^{2 \pi} \phi(R, \theta) e^{-i k \theta} d \theta
$$

Multiplying this last equation by $R^{k+1}$ and integrating with respect to $R$ from 0 to 1 we obtain the required result.

6. Determination of the power series of the mapping function. The coefficients of the power series

$$
f(z)=\frac{\mu}{\gamma(0)} \sum_{n=1}^{\infty} C_{n} z^{n}
$$

can be determined by differentiating twice both (20) and (23).

$$
\frac{\gamma(0)}{\mu} f^{\prime}(z)=\exp \left(2 \sum_{1}^{\infty} A_{k} z^{k}\right)=\sum_{1}^{\infty} n C_{n} z^{n-1},
$$

and

$$
2 \exp \left(2 \sum_{1}^{\infty} A_{k} z^{k}\right) \cdot \sum_{1}^{\infty} k A_{k} z^{k-1}=\sum_{1}^{\infty} n(n-1) C_{n} z^{n-2}
$$


Substituting the first factor from (24) we get

$$
2\left(\sum_{1}^{\infty} n C_{n} z^{n-1}\right)\left(\sum_{1}^{\infty} k A_{k} z^{k-1}\right)=\sum_{1}^{\infty} n(n-1) C_{n} z^{n-2} .
$$

Equating corresponding coefficients we obtain

$$
2 \sum A_{m} C_{n}=j(j-1) C_{j},
$$

where on the left $m$ and $n$ take the values $1,2, \ldots j-1$, their sum being always $j$. Hence

$$
C_{j}=\frac{2}{j(j-1)} \sum_{\nu=1}^{j-1} \nu(j-\nu) A_{j-\nu} C_{\nu}, \quad j=2,3, \ldots
$$

Together with $C_{1}=1$ this recursion formula determines all the $C_{j}$ 's from the $A_{k}$ 's.

7. An integral representation of $\log f^{\prime}(z)$. Instead of using a series we can express our mapping function also by an integral.

Starting from (20)

$$
\log \left[\frac{\gamma(0)}{\mu} f^{\prime}(z)\right]=2 \sum_{k=1}^{\infty} A_{k} z^{k},
$$

we replace on the right the $A_{k}$ 's by their values (19) and interchange formally summation and integration:

$$
\log \left[\frac{\gamma(0)}{\mu} f^{\prime}(z)\right]=-\frac{2}{\pi} \int_{0}^{1} \int_{0}^{2 \pi} \varphi(R, \theta) \sum_{k=1}^{\infty}(k+1)\left(z R e^{-i \theta}\right)^{k} R d R d \theta .
$$

Putting $q=z R e^{-i \theta}$ the series on the right is obtained by differentiating the geometric series

$$
\sum_{k=1}^{\infty} q^{k+1}
$$

with respect to $q$. Hence

$$
\log \left[\frac{\gamma(0)}{\mu} f^{\prime}(z)\right]=\frac{2}{\pi} \int_{0}^{1} \int_{0}^{2 \pi} \varphi(R, \theta)\left[1-\frac{1}{\left(1-z R e^{-i \bar{\theta}}\right)^{2}}\right] R d R d \theta .
$$

For $|z| \leqslant r_{0}<1$ and $R \leqslant 1$ we have $|q| \leqslant r_{0}<1$; consequently the above series is uniformly convergent. For $|z|<1$ the double integral in (25) is a proper integral of a continuous function; for $|z|=1$, however, it does not exist in general, not even as an improper integral for in this case

$$
\left|1-z R e^{-i \theta}\right|=\left|z-R e^{i \theta}\right| \text {. }
$$

Equation (25) may be transformed in the following way: Let

$$
e^{i \theta}=\zeta, \quad \varphi(R, \theta)=\psi(R, \zeta) .
$$

Then, since the function $\zeta \psi(R, \zeta)$ is defined continuously for $0 \leqslant R \leqslant 1$ and $|\zeta|=1$, we can form the expression

$$
\chi(R, t)=\frac{1}{2 \pi i} \oint \frac{\zeta \psi(R, \zeta)}{\zeta-t} d \zeta
$$


where the path of integration is the unit circle of the $\zeta$-plane. For $|t|<1$ this function is analytic in $t$, and we have

$$
\frac{\partial \chi}{\partial t}(R, t)=\frac{1}{2 \pi i} \oint \frac{\zeta \psi(R, \zeta)}{(\zeta-t)^{2}} d \zeta
$$

Transforming the integral in (24) and using (25) and (26) we obtain

$$
\log \left[\frac{\gamma(0)}{\mu} f^{\prime}(z)\right]=4 \int_{0}^{1}\left[\frac{\partial \chi}{\partial t}(R, 0)-\frac{\partial \chi}{\partial t}(R, z R)\right] R d R .
$$

One of the integrations is now relegated to the complex integration (26), which may be simpler.

For instance, let us make the further assumption about $\psi$, that $\zeta \psi(R, \zeta)$ can be extended into the interior of the unit circle of the $\zeta$-plane as a regular analytic function of $\zeta$. Then the integration in (26) can be carried out by Cauchy's formula and yields:

$$
\chi(R, t)=t \psi(R, t) .
$$

Hence we have from (28):

$$
\log \left[\frac{\gamma(0)}{\mu} f^{\prime}(z)\right]=4 \int_{0}^{1}\left[\psi(R, 0)-\psi(R, z R)-z R \frac{\partial \psi}{\partial \zeta}(R, z R)\right] R d R .
$$

It is remarkable that the minimum value of $I$ can be expressed by means of the function (26).

Let

$$
\chi(R, t)=\sum_{k=0}^{\infty} b_{k}(R) t^{k}
$$

Then it is easily seen from (17) that

$$
\begin{aligned}
I_{\min } & =2 \pi\left\{\int_{0}^{1}\left|b_{1}(R)\right|^{2} R d R-2\left|\int_{0}^{1} b_{1}(R) R d R\right|^{2}\right\} \\
& +4 \pi \sum_{k=1}^{\infty}\left\{\int_{0}^{1}\left|b_{k+1}(R)\right|^{2} R d R-2(k+1)\left|\int_{0}^{1} b_{k+1}(R) R^{k+1} d R\right|^{2}\right\} .
\end{aligned}
$$

8. Example. If the function $\varphi$ does not depend on $\theta$ the above-mentioned condition about the extension of $\zeta \psi$ is satisfied. Hence (29) is applicable and yields

$$
\log \left[\frac{\gamma(0)}{\mu} f^{\prime}(z)\right]=0, \quad f(z)=\frac{\mu}{\gamma(0)} \cdot z .
$$

Let now $D$ be a circular domain on a sphere. Using stereographic projection from one of the endpoints of the diameter perpendicular to the circle's plane, the line-element, and therefore also the function $\varphi$, obviously do not depend on $\theta$. Consequently equation (31) holds, which means (assuming the scale to be 1 at the centre): For a circular domain on a sphere the conformal mapping with least distortion is its symmetrical stereographic projection. 
9. Unsolved problems. In conclusion we mention three natural questions which we leave unanswered.

I. We have not studied the problem of the best choice of the point $M$ on the surface ("the centre of the map") whose image is $z=0$. Another choice $M_{1}$ instead of $M$ would lead to another normal isothermic system $z_{1}=x_{1}+i y_{1}$, mapping $D$ again on the unit circle. Consequently

$$
z_{1}=L(z)=e^{i \tau} \frac{z-\alpha}{\bar{\alpha} z-1}, \quad 0 \leqslant \tau<2 \pi, \quad|\alpha|<1,
$$

where $L(z)$ is a linear function mapping the unit circle onto itself. $M_{1}$ has in the old system the coordinates $z=L^{-1}(0)=\alpha$. For the line-element of $D$ we have

$$
d s=\frac{|d z|}{\gamma(z)}=\frac{\left|d z_{1}\right|}{\gamma_{1}\left(z_{1}\right)}=\frac{\left|L^{\prime}(z)\right||d z|}{\gamma_{1}[L(z)]},
$$

and therefore

$$
\gamma_{1}\left(z_{1}\right)=\left|L^{\prime}(z)\right| \gamma(z), \quad \varphi_{1}\left(z_{1}\right)=\log \gamma_{1}\left(z_{1}\right)=\log \left|L^{\prime}(z)\right|+\varphi(z) .
$$

Substituting this in $I_{\min }(30)$ we obtain a function of the complex quantity $\alpha$, which we have to minimize by a suitable choice of $\alpha$. The existence of such a minimum (under the side condition $|\alpha|<1$ ) seems difficult to prove in general.

II. For which regions $D$ will our map become a schlicht domain? A general answer to this question is difficult; we can hope that for not too large regions which are not too much curved our map will be schlicht, and perhaps more precise sufficient conditions can be found.

III. Conformal mapping in the large: Given two conformally equivalent closed surfaces find the mapping with least distortion (in some sense). Since the family of all the conformal mappings in this case depends on a finite number of parameters our problem reduces to a minimum problem instead of a variational problem. But even so the existence of a best map is not obvious.

\section{Reference}

1. Tchebycheff, Sur la construction des cartes géographiques; Oeuvres, tome I, pp. 233-236, 239-247.

\section{University of Alberta}

